\title{
Politique
}

Politique

\section{Les relations de pouvoir dans les lois : comparaisons entre les gouvernements du Québec de 1944 à 1985 de Vincent Lemieux, Sainte-Foy, Presses de l'université Laval et Institut d'administration publique du Canada, 1991, 247 p.}

\section{André Blais}

Numéro 21, hiver 1992

URI : https://id.erudit.org/iderudit/040717ar

DOI : https://doi.org/10.7202/040717ar

Aller au sommaire du numéro

Éditeur(s)

Société québécoise de science politique

ISSN

0711-608X (imprimé)

1918-6584 (numérique)

Découvrir la revue

Citer ce compte rendu

Blais, A. (1992). Compte rendu de [Les relations de pouvoir dans les lois : comparaisons entre les gouvernements du Québec de 1944 à 1985 de Vincent Lemieux, Sainte-Foy, Presses de l'université Laval et Institut d'administration publique du Canada, 1991, 247 p.] Politique, (21), 159-162.

https://doi.org/10.7202/040717ar d'utilisation que vous pouvez consulter en ligne.

https://apropos.erudit.org/fr/usagers/politique-dutilisation/ 
Les relations de pouvoir dans les lois : comparaisons entre les gouvernements du Québec de 1944 a 1985 de Vincent Lemieux, Sainte-Foy, Presses de I'université Laval et Institut d'administration publique du Canada, 1991, 247 p.

Vincent Lemieux nous propose une analyse des 2745 lois d'intérêt public sanctionnées au Québec de 1945 à 1985. Chaque loi est étudiée sous les angles suivants : sujet, objet, secteur d'intervention, nombre d'articles, enjeu, modalités de pouvoir. Par rapport à chacune de ces caractéristiques, l'auteur examine les variations d'une législature à une autre, d'un gouvernement à un autre et d'un parti à un autre.

La présentation est claire et succincte. En plus d'une introduction et d'une conclusion, d'une petite recension des écrits et d'une présentation de la méthode, un court chapitre, d'environ quinze pages, est consacré à chaque 
caractéristique, laquelle est mesurée à l'aide de quelques indicateurs; ainsi, la complexité sera évaluée au nombre d'articles, de relations de pouvoir impliquées, d'enjeux de contrôle, d'acteurs différents engagés... Pour chaque indicateur retenu, l'auteur présente les grandes tendances et les variations entre législatures, gouvernements et partis, et discute brievement la signification des résultats obtenus. Suivent deux annexes, la première sur la grille d'analyse et la seconde listant toutes les lois. Cela fait un petit livre de $\mathbf{1 5 0}$ pages (excluant la liste des lois).

Parmi les nombreuses observations de l'auteur, relevons les suivantes :

- Le nombre annuel moyen de lois n'a pas augmenté de façon constante.

- La complexité des lois augmente de façon constante d'un gouvernement de parti à un autre. La hausse procède par paliers, l'arrivée d'un nouveau gouvernement étant accompagnée d'une montée de la complexité, qui se stabilise jusqu'à l'arrivée d'un autre gouvernement.

- Plus de $40 \%$ des lois se situent dans la mission économique, et les gouvernements liberraux y sont plus actifs que ceux de I'Union nationale et du Parti québécois.

- Le gouvernement du Parti québécois est le plus "contrôleur", et le premier de l'Union nationale le moins.

- Les acteurs centraux (appareils politiques et administratifs) prédominent dans les lois, et de façon plus particulière sous les gouvernements libéraux.

Lemieux dégage en conclusion les traits marquants des différents partis. Le gouvernement du Parti québécois est celui qui se démarque le plus nettement : c'est le plus réformiste. Les gouvernements libéraux se caractérisent par leur sensibilité économique et leur propension à centraliser. Les gouvernements de l'Union nationale sont moins marqués, surtout le second (1966-1969) qui dénote peu d'originalité.

Les relations de pouvoir dans les lois a deux grands mérites. Dès le début, Lemieux fait le constat d'une absence 
totale d'étude méthodique du contenu des lois. Manifestement, cet ouvrage vient combler une lacune criante. Qu'il le fasse de façon incomplète et, à certains égards, insatisfaisante, j'en conviens. Mais Lemieux innove, et l'innovation n'est pas gratuite. II faut bien reconnaitre que les lois sont au cœur même de ce que produit l'État et qu'il importe de développer des grilles d'analyse systématique. En autant que je sache, c'est la première étude systématique des lois, au Québec ou ailleurs. On ne peut qu'espérer que la voie ainsi ouverte sera poursuivie et perfectionnée par d'autres. Le livre de Lemieux apporte également une contribution au niveau de la compréhension de l'État et des partis québécois. On n'y trouvera pas de grandes découvertes renversantes. Non, plus simplement, un certain nombre d'observations intéressantes, qui ajoutent un éclairage utile a la compréhension des choses.

Les relations de pouvoir dans les lois comporte deux lacunes principales à mon avis; la première, au niveau de l'interprétation des tendances. Sur un certain nombre d'indicateurs, Lemieux observe une tendance lourde sur l'ensemble de la période lla hausse de la complexité, par exemple). Cela pose un problème sérieux quand il s'agit ensuite de comparer les gouvernements et les partis. De façon plus concrète, le gouvernement du Parti québécois est-il différent du Parti libéral parce qu'il l'a suivi dans le temps ou parce que c'est un parti différent? Pour répondre de façon satisfaisante a cette question, il aurait fallu estimer la tendance lourde et déterminer si les écarts entre partis subsistent une fois celle-ci prise en compte.

La deuxième lacune est l'incapacité ou le refus de Lemieux de relier ses travaux a la littérature pertinente. L'étude peut être rattachée à deux créneaux : la littérature sur les politiques gouvernementales d'une part, et celle sur la politique québécoise d'autre part. Par rapport au premier créneau, on aimerait bien savoir en quoi l'image de l'État qui ressort de l'analyse des lois rejoint ou se démarque de celle véhiculée par les principaux courants théoriques. On $n$ 'apprendra rien à ce sujet, comme si la communication n'était possible qu'à l'intérieur d'une même perspective 
théorique et méthodologique. Par rapport au terrain québécois, les interprétations de la Révolution tranquille et du Parti québécois, en particulier, foisonnent. Pourquoi ne pas avoir fait le tour de ces interprétations et indiqué lesquelles semblent le mieux confirmées par les tendances observées au niveau des lois? L'auteur a beau annoncer (p. 127) qu'il se livre «à l'occasion» à l'exercice; en fait, la discussion de cette littérature est mince et plutôt superficielle.

Malgré ces lacunes, il faut lire ce petit livre. Ceux qui s'intéressent à l'analyse des politiques y verront une œuvre innovatrice, qui ouvre une nouvelle piste de recherche. Ceux qui s'intéressent a la politique québécoise auront droit à plusieurs observations utiles sur les gouvernements du Québec de 1944 à 1985.

André Blais Université de Montréal 